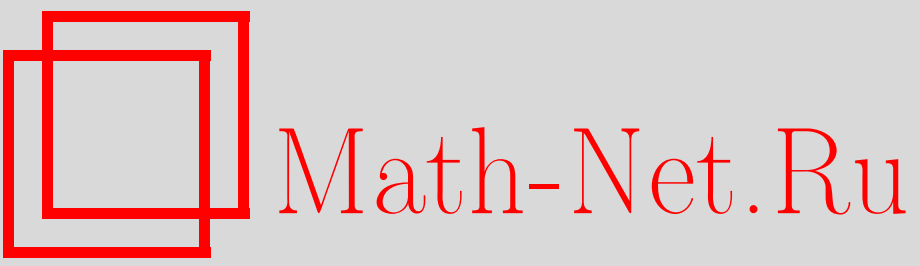

Ю. В. Павлов, $n$-Волновая процедура и размерная регуляризация для скалярного поля в однородном изотропном пространстве, ТМФ, 2004, том 138, номер 3, 453-467

DOI: https://doi.org/10.4213/tmf37

Использование Общероссийского математического портала Math-Net.Ru подразумевает, что вы прочитали и согласны с пользовательским соглашением

http://www.mathnet.ru/rus/agreement

Параметры загрузки:

IP: 18.234 .197 .8

26 апреля 2023 г., 17:31:57 


\title{
n-ВОЛНОВАЯ ПРОЦЕДУРА И РАЗМЕРНАЯ РЕГУЛЯРИЗАЦИЯ ДЛЯ СКАЛЯРНОГО ПОЛЯ В ОДНОРОДНОМ ИЗОТРОПНОМ ПРОСТРАНСТВЕ
}

\begin{abstract}
Получены выражения для вакуумных средних тензора энергии-импульса скалярного поля с произвольной связью с кривизной в $N$-мерном однородном изотропном пространстве для вакуума, определяемого методом диагонализации гамильтониана. Представлено обобщение $n$-волновой процедуры для $N$-мерного однородного изотропного пространства-времени. С использованием размерной регуляризации исследована геометрическая структура величин, вычитаемых из вакуумного тензора энергии-импульса в соответствии с $n$-волновой процедурой. Показано, что геометрические структуры первых трех вычитаний в $n$-волновой процедуре и в методе эффективного действия совпадают. Показано, что все вычитания $n$-волновой процедуры в 4 - и в 5 -мерных однородных изотропных пространствах соответствуют перенормировке констант затравочного гравитационного лагранжиана.
\end{abstract}

Ключевые слова: скалярное поле, квантовая теория в искривленном пространстве, перенормировка, размерная регуляризация.

\section{1. ВВЕДЕНИЕ}

Квантовая теория поля в искривленном пространстве-времени интенсивно развивается начиная с 70-х годов прошедшего столетия (см. монографии [1], [2]) и имеет важные приложения в космологии и астрофизике. В частности, эффект рождения частиц гравитационным полем из вакуума может быть использован для объяснения наблюдаемой плотности видимой и темной материи [3]. Сушествуют модели, объясняюшие наблюдаемое ускорение расширения Вселенной эффектами квантовой теории поля в искривленном пространстве [4].

При исследовании влияния квантованного поля на метрику пространства-времени необходимо вычислять вакуумные средние тензора энергии-импульса (ТЭИ), которые являются источниками гравитационного поля, в соответствии с полуклассическим подходом. Поскольку вакуумные средние ТЭИ представляются расходящимися выражениями, необходима перенормировка. Сушествуют различные способы устранения расхо-

* Институт проблем машиноведения РАН, Санкт-Петербург, Россия. E-mail: pavlov@ipme.ru 
димостей вакуумного ТЭИ (см. обзоры в [1], [2]). Для однородных изотропных пространств широко используются $n$-волновая процедура, предложенная в работе [5], и эквивалентная ей схема вычитаний $[6]$, называемая адиабатической регуляризацией. $\mathrm{C}$ их применением проведено большое количество расчетов (для скалярного поля с конформной связью с кривизной см. [1], [2]; в случае произвольной связи см., например, [7], [8]). Для строгого обоснования полученных выражений для вакуумного ТЭИ необходимо дать интерпретацию используемых схем вычитаний в терминах перенормировок констант затравочного лагранжиана. Для скалярного поля с произвольной связью с кривизной геометрическая структура бесконечных вычитаний адиабатической процедуры в однородном изотропном пространстве была установлена в работе [9]. Однако геометрическая структура конечных контрчленов и их интерпретация в терминах перенормировок оставались невыясненными. Для пространства-времени с плоскими пространственными сечениями полная геометрическая структура контрчленов $n$-волновой процедуры была определена с использованием размерной регуляризации в работах [10], [11]. В [11] было показано, что все вычитания $n$-волновой процедуры в четырехмерном пространстве-времени с плоскими пространственными сечениями соответствуют перенормировке констант затравочного размерно регуляризованного гравитационного лагранжиана.

Исследованию геометрической структуры контрчленов $n$-волновой процедуры в случае неплоских пространственных сечений и сравнению с результатами, получаемыми из размерно регуляризованного эффективного действия, посвящена данная работа. Отметим, что вычисления для $N$-мерного пространства-времени, необходимые в данной работе в связи с проведением размерной регуляризации, могут представлять и самостоятельный интерес для исследования эффектов квантовой теории в искривленном пространстве-времени в многомерных моделях.

В настоящей работе рассматривается комплексное скалярное поле с произвольной связью с кривизной в $N$-мерном пространстве-времени с однородными изотропными пространственными сечениями. В разделе 2 приведена геометрическая структура выражений, вычитаемых из вакуумного ТЭИ в методе эффективного действия. В разделе 3 получены выражения для вакуумных средних ТЭИ в однородном изотропном пространстве для вакуума, определенного в соответствии с методом диагонализации гамильтониана. В разделе 4 дано обобшение для $N$-мерного однородного изотропного случая $n$-волновой процедуры и приведены первые три контрчлена, которые исчерпьвают все контрчлены в размерностях $N=4,5$. С помошью размерной регуляризации исследуется геометрическая структура контрчленов $n$-волновой процедуры. В разделе 5 сформулированы основные результаты работы. В приложении А приведены некоторые формулы для собственных функций оператора Лапласа-Бельтрами в $(N-1)$-мерном пространстве постоянной кривизны, используемые при вычислении вакуумных средних ТЭИ. В приложении Б даны выражения для встречаюшихся в работе геометрических величин в $N$-мерном однородном изотропном пространстве-времени.

В статье используется система единиц, в которой $\hbar=c=1$. Знаки тензора кривизны и тензора Риччи выбраны так, что $R_{j k l}^{i}=\partial_{l} \Gamma_{j k}^{i}-\partial_{k} \Gamma_{j l}^{i}+\Gamma_{n l}^{i} \Gamma_{j k}^{n}-\Gamma_{n k}^{i} \Gamma_{j l}^{n}, R_{i k}=R_{i l k}^{l}$, где $\Gamma_{j k}^{i}$ - символы Кристоффеля. 
$n$-ВОЛНОВАЯ ПРОЦЕДУРА И РАЗМЕРНАЯ РЕГУЛЯРИЗАЦИЯ

\section{2. ГЕОМЕТРИЧЕСКАЯ СТРУКТУРА КОНТРЧЛЕНОВ В МЕТОДЕ ЭФФЕКТИВНОГО ДЕЙСТВИЯ}

Рассмотрим комплексное скалярное поле $\varphi(x)$ массы $m$ с уравнением движения

$$
\left(\nabla^{i} \nabla_{i}+\xi R+m^{2}\right) \varphi(x)=0
$$

соответствующим лагранжиану

$$
L(x)=\sqrt{|g|}\left[g^{i k} \partial_{i} \varphi^{*} \partial_{k} \varphi-\left(m^{2}+\xi R\right) \varphi^{*} \varphi\right]
$$

где $\nabla_{i}$ - ковариантные производные в метрике $g_{i k}, g=\operatorname{det}\left(g_{i k}\right), R$ - скалярная кривизна, $\xi$ - константа связи с кривизной. В пространстве-времени размерности $N$ при $\xi=$ $\xi_{\mathrm{c}} \equiv(N-2) /[4(N-1)]$ (конформная связь) и $m=0$ уравнение $(1)$ конформно-инвариантно $\left(\xi_{\mathrm{c}}=1 / 6\right.$ при $\left.N=4\right)$.

Метрический ТЭИ, получаемый варьированием действия по метрике, имеет вид (cM. [12])

$$
T_{i k}=\partial_{i} \varphi^{*} \partial_{k} \varphi+\partial_{k} \varphi^{*} \partial_{i} \varphi-g_{i k}|g|^{-1 / 2} L(x)-2 \xi\left(R_{i k}+\nabla_{i} \nabla_{k}-g_{i k} \nabla^{j} \nabla_{j}\right) \varphi^{*} \varphi .
$$

Выражения для вакуумных средних ТЭИ являются расходяшимися. В пространствевремени с метрикой обшего вида для анализа геометрической структуры расходимостей удобно использовать размерно регуляризованное эффективное действие. Для комплексного скалярного поля $\varphi(x)$ с уравнением движения (1) однопетлевое эффективное действие может быть записано в виде (см. [2], [13])

$$
S_{\text {eff }}=\int L_{\text {eff }}(x) \sqrt{|g|} d^{N} x
$$

где

$$
\begin{gathered}
L_{\mathrm{eff}}(x)=(4 \pi)^{-N / 2}\left(\frac{M}{m}\right)^{2 \varepsilon} \sum_{j=0}^{\infty} a_{j}(x) m^{N_{0}-2 j} \Gamma\left(j-\frac{N}{2}\right), \\
a_{0}(x)=1, \quad a_{1}(x)=\left(\frac{1}{6}-\xi\right) R \\
a_{2}(x)=\frac{1}{180} R_{l m p q} R^{l m p q}-\frac{1}{180} R_{l m} R^{l m}-\frac{1}{6}\left(\frac{1}{5}-\xi\right) \nabla^{l} \nabla_{l} R+\frac{1}{2}\left(\frac{1}{6}-\xi\right)^{2} R^{2},
\end{gathered}
$$

$N$ - размерность пространства-времени, которая рассматривается как переменная, аналитически продолженная в комплексную плоскость, $\varepsilon$ - комплексный параметр, $M-$ константа размерности массы [14], введенная для сохранения обычной размерности $L_{\text {eff }}$ (длина) $)^{-N_{0}}$ в случае $N=N_{0}-2 \varepsilon, \Gamma(z)$-гамма-функция.

Первые $\left[N_{0} / 2\right]+1$ слагаемых в (5) исключаются для получения перенормированного лагранжиана $L_{\text {eff }}([b]$ обозначает целую часть числа $b)$. Варьированием по $g_{i k}$ 
соответствуюших $j=0,1$ слагаемых в эффективном действии получим вычитаемые из вакуумного ТЭИ члены

$$
\begin{aligned}
T_{i k, \varepsilon}[0] & =-\frac{m^{N_{0}}}{2^{N_{0} \pi^{N_{0} / 2}}}\left(\frac{4 \pi M^{2}}{m^{2}}\right)^{\varepsilon} \Gamma\left(\varepsilon-\frac{N_{0}}{2}\right) g_{i k}, \\
T_{i k, \varepsilon}[1] & =\frac{m^{N_{0}-2}}{2^{N_{0}-1} \pi^{N_{0} / 2}}\left(\frac{1}{6}-\xi\right)\left(\frac{4 \pi M^{2}}{m^{2}}\right)^{\varepsilon} \Gamma\left(1-\frac{N}{2}\right) G_{i k}= \\
& =\frac{m^{N_{0}-2}}{2^{N_{0}-1} \pi^{N_{0} / 2}}\left(\frac{4 \pi M^{2}}{m^{2}}\right)^{\varepsilon}\left[\frac{-\Gamma(3-N / 2)}{3(N-1)(N-2)}+\Delta \xi \Gamma\left(1-\frac{N}{2}\right)\right] G_{i k},
\end{aligned}
$$

где $G_{i k}=R_{i k}-R g_{i k} / 2-$ тензор Эйнштейна, $\Delta \xi \equiv \xi_{\mathrm{c}}-\xi$.

Найдем член $T_{i k, \varepsilon}[2]$, соответствуюший вычитанию из $L_{\mathrm{eff}}$ слагаемого с $j=2$. Для анализа случая однородных изотропных пространств удобно переписать (7) следуюшим образом:

$$
\begin{aligned}
a_{2}(x)= & \frac{N-6}{720(N-3)}\left(R_{l m p q} R^{l m p q}-4 R_{l m} R^{l m}+R^{2}\right)+\frac{(N-2) C_{l m p q} C^{l m p q}}{240(N-3)}- \\
& -\frac{1}{6}\left(\frac{1}{5}-\xi\right) \nabla^{l} \nabla_{l} R+\left(\frac{(N-4)(N-6)}{480(N-1)^{2}}+\frac{\Delta \xi(4-N)}{12(N-1)}+\frac{(\Delta \xi)^{2}}{2}\right) R^{2},
\end{aligned}
$$

где

$$
C_{i k l m}=R_{i k l m}+\frac{2}{N-2}\left(R_{m[i} g_{k] l}-R_{l[i} g_{k] m}\right)+\frac{2 R g_{l[i} g_{k] m}}{(N-1)(N-2)}
$$

- конформный тензор Вейля, квадратные скобки в индексах означают антисимметризацию: $A_{n[i} B_{k] m}=\left(A_{n i} B_{k m}-A_{n k} B_{i m}\right) / 2$. Используя (10) и варьируя по $g_{i k}$ соответствуюшее $j=2$ слагаемое в эффективном действии, получим $T_{i k, \varepsilon}[2]$ в следующем виде:

$$
\begin{aligned}
T_{i k, \varepsilon}[2]= & \frac{m^{N_{0}-4}}{(4 \pi)^{N_{0} / 2}}\left(\frac{4 \pi M^{2}}{m^{2}}\right)^{\varepsilon}\left[\frac{(N-6) E_{i k}}{360(N-3)} \Gamma\left(2-\frac{N}{2}\right)+\frac{(N-2) W_{i k}}{120(N-3)} \Gamma\left(2-\frac{N}{2}\right)+\right. \\
& \left.+\left(\frac{\Gamma(4-N / 2)}{60(N-1)^{2}}+\Delta \xi \frac{\Gamma(3-N / 2)}{3(N-1)}+(\Delta \xi)^{2} \Gamma\left(2-\frac{N}{2}\right)\right){ }^{(1)} H_{i k}\right]
\end{aligned}
$$

где

$$
\begin{aligned}
{ }^{(1)} H_{i k} & =\frac{1}{\sqrt{|g|}} \frac{\delta}{\delta g^{i k}} \int R^{2} \sqrt{|g|} d^{N} x= \\
& =2\left(\nabla_{i} \nabla_{k} R-g_{i k} \nabla^{l} \nabla_{l} R\right)+2 R\left(R_{i k}-\frac{1}{4} R g_{i k}\right), \\
E_{i k} & =\frac{1}{\sqrt{|g|}} \frac{\delta}{\delta g^{i k}} \int\left(R_{l m p q} R^{l m p q}-4 R_{l m} R^{l m}+R^{2}\right) \sqrt{|g|} d^{N} x, \\
W_{i k} & =\frac{1}{\sqrt{|g|}} \frac{\delta}{\delta g^{i k}} \int C_{l m p q} C^{l m p q} \sqrt{|g|} d^{N} x .
\end{aligned}
$$


В четырехмерном пространстве-времени $E_{i k}=0$, так как в соответствии с теоремой Гаусса-Бонне

$$
\frac{\delta}{\delta g^{i k}} \int\left(R^{l m p q} R_{l m p q}-4 R^{l m} R_{l m}+R^{2}\right) \sqrt{|g|} d^{4} x=0 .
$$

Используя $(11),(13)$ и следуюшие формулы для вариационных производных от квадратичных по кривизне выражений (см. [15]):

$$
\begin{aligned}
{ }^{(2)} H_{i k}= & \frac{1}{\sqrt{|g|}} \frac{\delta}{\delta g^{i k}} \int R^{l m} R_{l m} \sqrt{|g|} d^{N} x=\nabla_{i} \nabla_{k} R-\nabla^{l} \nabla_{l} R_{i k}- \\
& -\frac{1}{2}\left(\nabla^{l} \nabla_{l} R+R^{l m} R_{l m}\right) g_{i k}+2 R^{l m} R_{l i m k}, \\
H_{i k}= & \frac{1}{\sqrt{|g|}} \frac{\delta}{\delta g^{i k}} \int R^{l m p q} R_{l m p q} \sqrt{|g|} d^{N} x=2 \nabla_{i} \nabla_{k} R-4 \nabla^{l} \nabla_{l} R_{i k}- \\
& -4 R_{i l} R_{k}^{l}+4 R^{l m} R_{l i m k}-\frac{1}{2} g_{i k} R_{l m p q} R^{l m p q}+2 R_{i l m p} R_{k}^{l m p},
\end{aligned}
$$

для $E_{i k}$ при произвольном $N$ можно получить (см. [13])

$$
E_{i k}=H_{i k}-4^{(2)} H_{i k}+{ }^{(1)} H_{i k}=2 C_{i l m p} C_{k}^{l m p}-\frac{g_{i k}}{2} C_{l m p q} C^{l m p q}-(N-4)^{(3)} H_{i k},
$$

где

$$
\begin{aligned}
{ }^{(3)} H_{i k}= & \frac{4}{N-2} C_{i l k m} R^{l m}+\frac{2(N-3)}{(N-2)^{2}}\left[2 R_{i l} R_{k}^{l}-\frac{N}{N-1} R R_{i k}-\right. \\
& \left.-g_{i k}\left(R_{l m} R^{l m}-\frac{N+2}{4(N-1)} R^{2}\right)\right] .
\end{aligned}
$$

В конформно-плоском случае $\left(C_{i k l m}=0\right)$ тензор ${ }^{(3)} H_{i k}$ ковариантно сохраняется, что следует из (16) при $N \neq 4$ и непосредственно проверяется для $N=4$. Тензор ${ }^{\left({ }^{3)}\right.} H_{i k}$, предложенный в статье [13], является обобщением соответствуюшего тензора, введенного в работе [16] для конформно-плоского четырехмерного случая.

При $N \rightarrow 4$ произведение $E_{i k} \Gamma(2-(N / 2))$ для произвольной метрики пространства-времени имеет конечный предел, поскольку при аналитическом продолжении по размерности зависимость выражений от $N$ предполагается дробно-рациональной, $E_{i k}=0$ при $N=4$ и гамма-функция имеет в нуле полюс первого порядка. Таким образом, первое слагаемое в квадратных скобках в правой части (12) при $N \rightarrow 4$ всегда конечно. Если не вычитать это слагаемое при перенормировке ТЭИ, то при $N \rightarrow 4$ вакуумный ТЭИ будет конечным, однако эффективное действие при этом останется расходящимся и выражение для аномального следа вакуумного ТЭИ будет отличаться от стандартного. При дополнительной конечной перенормировке коэффициента при $R^{2}$ в $L_{\text {eff }}$ (обсуждение этого вопроса см. в [2], § 6.3) аномальный след для конформного скалярного поля в конформно-плоском пространстве может быть сделан нулевым. Такой результат имеет место, например, в работе [17], где для получения конечных значений вакуумного ТЭИ в однородном изотропном пространстве для конформного скалярного поля было использовано зависяшее от времени нормальное упорядочение операторов. 
Далее в соответствии со стандартным подходом мы сохраняем слагаемое с $E_{i k}$ в (12). В конформно-плоском случае, в частности в однородном изотропном пространстве-времени, учтя равенства $C_{i k l m}=0, W_{i k}=0$ и (16), получим следуюшее выражение для члена $T_{i k, \varepsilon}[2]$, вычитаемого из вакуумного ТЭИ в методе эффективного действия:

$$
\begin{aligned}
T_{i k, \varepsilon}[2]= & \frac{m^{N_{0}-4}}{(4 \pi)^{N_{0} / 2}}\left(\frac{4 \pi M^{2}}{m^{2}}\right)^{\varepsilon}\left[\frac{-{ }^{(3)} H_{i k}}{90(N-3)} \Gamma\left(4-\frac{N}{2}\right)+\right. \\
& \left.+\left(\frac{\Gamma(4-N / 2)}{60(N-1)^{2}}+\Delta \xi \frac{\Gamma(3-N / 2)}{3(N-1)}+(\Delta \xi)^{2} \Gamma\left(2-\frac{N}{2}\right)\right){ }^{(1)} H_{i k}\right] .
\end{aligned}
$$

\section{3. СКАЛЯРНОЕ ПОЛЕ В ОДНОРОДНОМ ИЗОТРОПНОМ ПРОСТРАНСТВЕ}

Метрику $N$-мерного однородного изотропного пространства-времени запишем в виде

$$
d s^{2}=g_{i k} d x^{i} d x^{k}=a^{2}(\eta)\left(d \eta^{2}-d l^{2}\right),
$$

где $d l^{2}=\gamma_{\alpha \beta} d x^{\alpha} d x^{\beta}$ - метрика $(N-1)$-мерного пространства постоянной кривизны $K=0, \pm 1$. Полная система решений уравнения (1) в метрике (19) может быть найдена в форме

$$
\varphi(x)=a^{-(N-2) / 2}(\eta) g_{\lambda}(\eta) \Phi_{J}(\mathbf{x}),
$$

где

$$
\begin{gathered}
g_{\lambda}^{\prime \prime}(\eta)+\Omega^{2}(\eta) g_{\lambda}(\eta)=0 \\
\Omega^{2}(\eta)=m^{2} a^{2}+\lambda^{2}-\Delta \xi a^{2} R \\
\Delta_{N-1} \Phi_{J}(\mathbf{x})=-\left(\lambda^{2}-\left(\frac{N-2}{2}\right)^{2} K\right) \Phi_{J}(\mathbf{x}),
\end{gathered}
$$

штрих означает производную по конформному времени $\eta, J$ - набор индексов (квантовых чисел), нумеруюших собственные функции оператора Лапласа-Бельтрами $\Delta_{N-1}$ в $(N-1)$-мерном пространстве. Отметим, что из неотрицательности собственных значений оператора $-\Delta_{N-1}$ следует неравенство $\lambda^{2}-((N-2) / 2)^{2} K \geqslant 0$.

В соответствии с методом диагонализации гамильтониана [1] (случай произвольного $\xi$ см. в [18]) функции $g_{\lambda}(\eta)$ должны удовлетворять следующим начальным условиям:

$$
g_{\lambda}^{\prime}\left(\eta_{0}\right)=i \Omega\left(\eta_{0}\right) g_{\lambda}\left(\eta_{0}\right), \quad\left|g_{\lambda}\left(\eta_{0}\right)\right|=\frac{1}{\sqrt{\Omega\left(\eta_{0}\right)}} .
$$

Для квантования разложим поле $\varphi(x)$ по полной системе решений $(20)$

$$
\varphi(x)=\int d \mu(J)\left[\varphi_{J}^{(+)} a_{J}^{(+)}+\varphi_{J}^{(-)} a_{J}^{(-)}\right]
$$

где

$$
\varphi_{J}^{(+)}(x)=\frac{a^{-(N-2) / 2}(\eta)}{\sqrt{2}} g_{\lambda}(\eta) \Phi_{J}^{*}(\mathbf{x}), \quad \varphi_{J}^{(-)}(x)=\left(\varphi_{J}^{(+)}(\mathbf{x})\right)^{*}
$$


и потребуем выполнения коммутационных соотношений

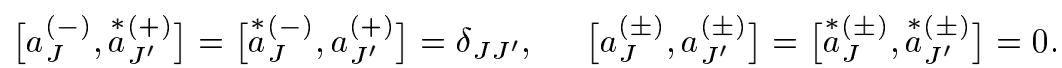

Средние от оператора ТЭИ по вакууму $|0\rangle$, уничтожаемому операторами $a_{J}^{(-)}, \stackrel{*}{a}_{J^{\prime}}^{(-)}$, удобно выражать через билинейные комбинации функций $g_{\lambda}$ и $g_{\lambda}^{*}$ :

$$
S=\frac{\left|g_{\lambda}^{\prime}\right|^{2}+\Omega^{2}\left|g_{\lambda}\right|^{2}}{4 \Omega}-\frac{1}{2}, \quad U=\frac{\Omega^{2}\left|g_{\lambda}\right|^{2}-\left|g_{\lambda}^{\prime}\right|^{2}}{2 \Omega}, \quad V=-\frac{d\left(g_{\lambda}^{*} g_{\lambda}\right)}{2 d \eta},
$$

которые в соответствии с (21) удовлетворяют системе дифференциальных уравнений

$$
S^{\prime}=\frac{\Omega^{\prime}}{2 \Omega} U, \quad U^{\prime}=\frac{\Omega^{\prime}}{\Omega}(1+2 S)-2 \Omega V, \quad V^{\prime}=2 \Omega U
$$

С учетом начальных условий $S\left(\eta_{0}\right)=U\left(\eta_{0}\right)=V\left(\eta_{0}\right)=0$, следуюших из (24), уравнения (28) могут быть записаны в виде системы интегральных уравнений Вольтерра

$$
\begin{aligned}
& U(\eta)+i V(\eta)=\int_{\eta_{0}}^{\eta} w\left(\eta_{1}\right)\left(1+2 S\left(\eta_{1}\right)\right) e^{2 i \Theta\left(\eta_{1}, \eta\right)} d \eta_{1}, \\
& S(\eta)=\frac{1}{2} \int_{\eta_{0}}^{\eta} d \eta_{1} w\left(\eta_{1}\right) \int_{\eta_{0}}^{\eta_{1}} w\left(\eta_{2}\right)\left(1+2 S\left(\eta_{2}\right)\right) \cos \left[2 \Theta\left(\eta_{2}, \eta_{1}\right)\right] d \eta_{2},
\end{aligned}
$$

где

$$
w(\eta)=\frac{\Omega^{\prime}(\eta)}{\Omega(\eta)}, \quad \Theta\left(\eta_{1}, \eta_{2}\right)=\int_{\eta_{1}}^{\eta_{2}} \Omega(\eta) d \eta .
$$

Подставляя разложение $(25)$ в (3) и используя (26), (27) и формулы (П.1)-(П.4) (см. приложение A), для вакуумных средних ТЭИ получим следующие (расходящиеся) выражения:

$$
\left\langle 0\left|T_{i k}\right| 0\right\rangle=\frac{B_{N}}{a^{N-2}} \int \tau_{i k} d \mu(\lambda)
$$

где $B_{N}=\left[2^{N-3} \pi^{(N-1) / 2} \Gamma((N-1) / 2)\right]^{-1}$, “спектральные плотности" $\tau_{i k}$ равны

$$
\begin{aligned}
\tau_{00}= & \Omega\left(S+\frac{1}{2}\right)+\Delta \xi(N-1)\left[c V+\left(c^{\prime}+(N-2) c^{2}\right) \frac{1}{\Omega}\left(S+\frac{1}{2} U+\frac{1}{2}\right)\right], \\
\tau_{\alpha \beta}= & \gamma_{\alpha \beta}\left\{\frac{\lambda^{2}}{(N-1) \Omega}\left(S+\frac{1}{2}\right)-\frac{\Omega^{2}-\lambda^{2}}{2(N-1) \Omega} U-\right. \\
& \left.-\Delta \xi\left[\left((N-1) c^{\prime}+(N-2) K\right) \frac{1}{\Omega}\left(S+\frac{1}{2} U+\frac{1}{2}\right)+2 \Omega U-(N-1) c V\right]\right\},
\end{aligned}
$$

$c \equiv a^{\prime} / a$. Мера интегрирования в (30) в четырехмерном пространстве-времени имеет вид (см. [1])

$$
\int d \mu(\lambda) \ldots= \begin{cases}\int_{0}^{\infty} d \lambda \lambda^{2} \ldots, & K=0,-1 \\ \sum_{\lambda=1}^{\infty} \lambda^{2} \ldots, & K=1 .\end{cases}
$$


В $N$-мерном случае

$$
\int d \mu(\lambda) \ldots=\int d \lambda f(\lambda) 2 B_{N}^{-1} \ldots
$$

где функция $f(\lambda)$ определена в (П.1) и в квазиевклидовом $(K=0)$ и сферическом $(K=1)$ случаях может быть записана соответственно в виде (П.6), (П.7).

Отметим, что выражения для вакуумных средних ТЭИ, представленные в работе [7], справедливы только для $N=4$, а в [1] они вычислены по вакуумному состоянию, не соответствующему (при $\xi \neq \xi_{\text {c }}$ и $R \neq 0$ ) методу диагонализации гамильтониана.

\section{4. n-ВОЛНОВАЯ ПРОЦЕДУРА}

В однородных изотропных пространствах для выгисления перенормированного вакуумного ТЭИ часто применяется $n$-волновая процедура, предложенная в работе [5]. В квазиевклидовом $(K=0)$ случае она заключается в следуюшем. Если вакуумные средние некоторого билинейного по полю оператора $A$ выражаются в виде

$$
\langle 0|A| 0\rangle=\int a(\lambda, m) d \lambda
$$

то соответствуюшее перенормированное значение получают регуляризацией вклада каждой моды (т.е. подынтегральной функции с данным $\lambda$ ). Для этого заменяют $\lambda \rightarrow$ $n \lambda, m \rightarrow n m$ и вычитают из $a(\lambda, m)$ члены асимптотического разложения по $1 / n$ при $n \rightarrow \infty$, обусловливающие расходимость интеграла (33). Такая процедура соответствует выгитанию вклада так называемых $n$-волн (подробнее см. [5] и [1], § 2.2). Данному методу устранения расходимостей эквивалентен метод адиабатической регуляризации, основанный на введении параметра адиабатичности изменения метрики и вычитании в (33) первых членов асимптотического разложения $a(\lambda, m)$ по обратным степеням этого параметра (см. [2])

В случае сферического $(K=1)$ и гиперболического $(K=-1) N$-мерного пространства-времени при применении $n$-волновой процедуры необходимо учесть отличие меры в интеграле (30) от квазиевклидова случая. Записав меру интегрирования в виде $d \mu(\lambda)=\sigma(\lambda) d \lambda$, примем как обобщение $n$-волновой процедуры для $N$-мерного однородного изотропного пространства-времени следуюшее выражение:

$$
\left\langle 0\left|T_{i k}\right| 0\right\rangle_{\text {ren }}=\frac{B_{N}}{a^{N-2}} \lim _{\Lambda \rightarrow \infty}\left[\int^{\Lambda} \tau_{i k} d \mu(\lambda)-\sum_{l=0}^{[N / 2]} \int_{0}^{\Lambda} \lambda^{N-2} a_{i k}[l] d \lambda\right]
$$

где

$$
a_{i k}[l]=\frac{1}{l !} \lim _{n \rightarrow \infty} \frac{\partial^{l}}{\partial\left(n^{-2}\right)^{l}}\left(\frac{\tau_{i k}(n \lambda, n m) \sigma(n \lambda)}{n^{N-1} \lambda^{N-2}}\right)
$$

Данное обобщение в четырехмерном случае совпадает со стандартным определением $n$-волновой процедуры. Функция $\sigma(\lambda)$ учитьвает отличие меры интегрирования от $\lambda^{N-2} d \lambda$ при $K \neq 0$ и $N \neq 4$. 
Найдем явный вид $a_{i k}[l]$. Для этого разложим $S, U, V$ (см. (27)) по обратным степеням $n$ при замене $\lambda \rightarrow n \lambda, m \rightarrow n m$ и $n \rightarrow \infty:$

$$
S=\sum_{k=1}^{\infty} n^{-k} S_{k}, \quad \ldots
$$

Используя последовательные итерации в интегральных уравнениях (29) и метод стационарной фазы, получим для первых отличных от нуля членов разложений

$$
\begin{aligned}
& V_{1}=W, \quad U_{2}=D W, \quad S_{2}=\frac{1}{4} W^{2}, \quad V_{3}=\frac{1}{2} W^{3}-D^{2} W-\frac{\omega}{2} D\left(\frac{q}{\omega^{3}}\right) \\
& U_{4}=\frac{3}{2} W^{2} D W-D^{3} W-D\left(\frac{\omega}{2} D\left(\frac{q}{\omega^{3}}\right)\right)+\frac{q}{2 \omega^{2}} D W \\
& S_{4}=\frac{3}{16} W^{4}+\frac{1}{4}(D W)^{2}-\frac{1}{2} W D^{2} W-\frac{1}{4} \omega W D\left(\frac{q}{\omega^{3}}\right)
\end{aligned}
$$

где

$$
q=\Delta \xi a^{2} R, \quad \omega=\left(m^{2} a^{2}+\lambda^{2}\right)^{1 / 2}, \quad W=\frac{\omega^{\prime}}{2 \omega^{2}}, \quad D=\frac{1}{2 \omega} \frac{d}{d \eta}
$$

Необходимо отметить, что при вычислении величин $S_{k}, U_{k}, V_{k}$, как и в [1], [5], [10], мы пренебрегаем зависящими от начального момента времени $\eta_{0}$ слагаемыми, т.е. ограничиваемся случаем контрчленов, локальных по времени $\eta$. В частности, нелокальные слагаемые отсутствуют, если предполагается, что первые $2[N / 2]$ производных от масштабного фактора $a(\eta)$ метрики обрашаются в нуль в начальный момент времени.

Используем (31), (35) и полагаем далее для меры $\sigma(\lambda) d \lambda$

$$
\sigma(\lambda)=\lambda^{N-2}+\alpha_{N} \lambda^{N-4}+\beta_{N} \lambda^{N-6}+\cdots
$$

где $\alpha_{N}, \beta_{N}, \ldots$ - функции от $N$ и $K$, обращающиеся в нуль при $N=4$, которые будут определены ниже. В результате приходим к следующим выражениям для $a_{i k}[l]$ :

$$
\begin{array}{ll}
a_{00}[0]=\tau_{00}[0]=\frac{\omega}{2}, & a_{\alpha \beta}[0]=\tau_{\alpha \beta}[0]=\gamma_{\alpha \beta} \frac{\lambda^{2}}{2(N-1) \omega}, \\
a_{i k}[1]=\tau_{i k}[1]+\frac{\alpha_{N}}{\lambda^{2}} \tau_{i k}[0], & a_{i k}[2]=\tau_{i k}[2]+\frac{\alpha_{N}}{\lambda^{2}} \tau_{i k}[1]+\frac{\beta_{N}}{\lambda^{4}} \tau_{i k}[0],
\end{array}
$$


где

$$
\begin{aligned}
\tau_{00}[1]= & \omega S_{2}+\Delta \xi(N-1)\left[c V_{1}+\frac{N-2}{4 \omega}\left(c^{2}-K\right)\right], \\
\tau_{\alpha \beta}[1]= & \frac{\gamma_{\alpha \beta}}{N-1}\left[\frac{\lambda^{2}}{\omega} S_{2}-\frac{m^{2} a^{2}}{2 \omega}\left(U_{2}+\frac{q}{2 \omega^{2}}\right)\right]+\gamma_{\alpha \beta} \Delta \xi\left[(N-1) c V_{1}-\right. \\
& \left.-2 \omega\left(U_{2}+\frac{q}{2 \omega^{2}}\right)+\frac{1}{4 \omega}\left(-4 \xi a^{2} R+(N-2)\left(c^{2}(N-1)+K(N-3)\right)\right)\right], \\
\tau_{00}[2]=\omega & \left(S_{4}+\frac{q}{4 \omega^{2}} U_{2}+\frac{q^{2}}{16 \omega^{4}}\right)+ \\
& +\Delta \xi(N-1)\left[c V_{3}+\frac{N-2}{2 \omega}\left(c^{2}-K\right)\left(S_{2}+\frac{1}{2} U_{2}+\frac{q}{4 \omega^{2}}\right)\right], \\
\tau_{\alpha \beta}[2]= & \gamma_{\alpha \beta}\left\{\frac { 1 } { N - 1 } \left[\frac{\lambda^{2}}{\omega}\left(S_{4}+\frac{q}{4 \omega^{2}} U_{2}+\frac{q^{2}}{16 \omega^{4}}\right)-\frac{m^{2} a^{2}}{2 \omega}\left(U_{4}+\frac{q^{2}}{4 \omega^{4}}+\right.\right.\right. \\
& \left.\left.+\frac{q}{\omega^{2}} S_{2}\right)\right]+\Delta \xi\left[(N-1) c V_{3}-2 \omega\left(U_{4}+\frac{q^{2}}{4 \omega^{4}}+\frac{q}{\omega^{2}} S_{2}\right)+\right. \\
& \left.\left.+\frac{1}{2 \omega}\left(-4 \xi a^{2} R+(N-2)\left(c^{2}(N-1)+K(N-3)\right)\right)\left(S_{2}+\frac{1}{2} U_{2}+\frac{q}{4 \omega^{2}}\right)\right]\right\} .
\end{aligned}
$$

Приведенные выражения исчерпывают все вычитания в размерностях $N=4,5$. При $N \geqslant 6$ появляются дополнительные контрчлены (для конформного скалярного поля при $K=0$ они приведены в работе [11]).

Перенормированный в соответствии с (34) вакуумный ТЭИ ковариантно сохраняется. Это доказывается с помощью выражений $(37)$ и равенств $\nabla^{i}\left(\tau_{i k} / a^{N-2}\right)=0$ и $\nabla^{i}\left(\tau_{i k}[l] / a^{N-2}\right)=0$, которые следуют из $(28),(31),(38)$.

Для выяснения геометрической структуры контрчленов $n$-волновой процедуры используем размерную регуляризацию, полагая, как и в разделе $2, N=N_{0}-2 \varepsilon$, где $\varepsilon$ - комплексный параметр. При вычислении интегралов в размерно регуляризованных контрчленах

$$
T_{i k, \varepsilon}[l]=\frac{B_{N}}{a^{N-2}}(M)^{2 \varepsilon} \int_{0}^{\infty} \lambda^{N-2} a_{i k, \varepsilon}[l] d \lambda,
$$

где $a_{i k, \varepsilon}[l]$ определяются по формулам $(37),(38)$ с заменой $N \rightarrow N_{0}-2 \varepsilon$, используем равенство

$$
\int_{0}^{\infty} x^{k}\left(1+x^{2}\right)^{-p} d x=\frac{\Gamma((k+1) / 2) \Gamma(p-(k+1) / 2)}{2 \Gamma(p)}
$$

и аналитическое продолжение выражения в его правой части. Результат для нулевого контрчлена совпадает с (8). Таким образом, как в методе эффективного действия, так и в $n$-волновой процедуре нулевое вычитание соответствует перенормировке космологической постоянной.

С учетом формул (П. 12), (П.14) (см. приложение Б) структура первого и второго вычитаний $n$-волновой процедуры в однородном изотропном пространстве-времени может 
быть получена добавлением $\Delta T_{i k, \varepsilon}[1]$ и $\Delta T_{i k, \varepsilon}[2]$ к $(9)$ и (18), соответственно, где

$$
\begin{aligned}
& \Delta T_{i k, \varepsilon}[1]=\frac{m^{N_{0}-2}}{2^{N_{0}-1} \pi^{N_{0} / 2}}\left(\frac{4 \pi M^{2}}{m^{2}}\right)^{\varepsilon} D_{i k}[1] \\
& \Delta T_{i k, \varepsilon}[2]=\frac{m^{N_{0}-4}}{(4 \pi)^{N_{0} / 2}}\left(\frac{4 \pi M^{2}}{m^{2}}\right)^{\varepsilon} D_{i k}[2] \text {, } \\
& D_{00}[1]=-\frac{1}{6} \Gamma\left(3-\frac{N}{2}\right)\left(K+\frac{24 \alpha_{N}}{(N-2)(N-3)(N-4)}\right) \text {, } \\
& D_{\alpha \beta}[1]=\gamma_{\alpha \beta} \frac{N-3}{6(N-1)} \Gamma\left(3-\frac{N}{2}\right)\left(K+\frac{24 \alpha_{N}}{(N-2)(N-3)(N-4)}\right) \text {, } \\
& D_{00}[2]=\frac{N-2}{36 a^{2}}\left\{\left[\Gamma\left(4-\frac{N}{2}\right)(N-4) c^{2}+\Delta \xi \Gamma\left(3-\frac{N}{2}\right) 6(N-1) \times\right.\right. \\
& \left.\times\left((N-2) K+(N-6) c^{2}\right)\right]\left[K+\frac{24 \alpha_{N}}{(N-2)(N-3)(N-4)}\right]+ \\
& \left.+\Gamma\left(4-\frac{N}{2}\right)\left(\frac{(5 N-8) K^{2}}{10}-\frac{576 \beta_{N}}{(N-2)(N-3)(N-4)(N-5)(N-6)}\right)\right\}, \\
& D_{\alpha \beta}[2]=\frac{\gamma_{\alpha \beta}(N-2)}{36 a^{2}(N-1)}\left\{\left[\Gamma\left(4-\frac{N}{2}\right)(N-4)\left((5-N) c^{2}-2 c^{\prime}\right)+\right.\right. \\
& +\Delta \xi \Gamma\left(3-\frac{N}{2}\right) 6(N-1) \times \\
& \left.\times\left((5-N)(N-2) K+(N-6)\left((5-N) c^{2}-2 c^{\prime}\right)\right)\right] \times \\
& \times\left[K+\frac{24 \alpha_{N}}{(N-2)(N-3)(N-4)}\right]+\Gamma\left(4-\frac{N}{2}\right) \frac{(5-N)(5 N-8)}{10} \times \\
& \left.\times\left(K^{2}-\frac{5760 \beta_{N}}{(N-2)(N-3)(N-4)(N-5)(N-6)(5 N-8)}\right)\right\} \text {. }
\end{aligned}
$$

Следовательно, для того чтобы геометрическая структура первого и второго вычитаний в $n$-волновой процедуре и в методе эффективного действия совпадали, необходимо для произвольного $N$ выполнение тех же равенств (П.9), которые получены в приложении А для целых значений $N$ и $k=0,1$. При $N=4$ имеем $\alpha_{N}=\beta_{N}=0$, что соответствует (32). Таким образом, сушествует такое продолжение по размерности меры интегрирования в импульсном пространстве $\{\lambda\}$, что геометрические структуры всех вычитаний в $n$-волновой процедуре для четырехмерного однородного изотропного пространства-времени и в методе эффективного действия совпадают.

Вычисленные контрчлены имеют чисто геометрическую структуру (см. (8), (9), (18)) и могут быть представлены как вариационные производные по метрике от геометрических величин. Поэтому можно построить такой лагранжиан для гравитационного поля, что добавление этих контрчленов будет приводить к перенормировке его параметров (обсуждение этого подхода см. в [19], § 6.11, а также в [2], § 6.1). Поскольку контрчлены содержат квадратичные по кривизне выражения, то такой затравочный гравитационньй лагранжиан, очевидно, не будет совпадать со стандартным эйнштейновским. Наличие квадратичных по кривизне выражений указывает на то, что при квантовании по- 
лей в искривленном пространстве неизбежен выход за рамки эйнштейновской теории гравитации ([1], § 8.4). Значения перенормированных констант должны определяться из эксперимента, и возможно, как указано, например, в [1], что ренормированные константы при квадратичных членах равны нулю.

Используя формулы (8), (9), (18) и

$$
R^{l m p q} R_{l m p q}-4 R^{l m} R_{l m}+R^{2}=C_{l m p q} C^{l m p q}-\frac{4(N-3)}{N-2}\left(R_{l m} R^{l m}-\frac{N R^{2}}{4(N-1)}\right)
$$

можно сделать следующий вывод. Первые три вычитания $n$-волновой процедуры в $N$-мерном однородном изотропном пространстве-времени соответствуют перенормировке космологической и гравитационной постоянных и параметров при квадратичных по кривизне слагаемых в затравочном гравитационном лагранжиане вида

$$
L_{\mathrm{gr}, \varepsilon}=\sqrt{|g|}\left[\frac{1}{16 \pi G_{\varepsilon}}\left(R-2 \Lambda_{\varepsilon}\right)+\alpha_{\varepsilon}\left(R^{i k} R_{i k}-\frac{N R^{2}}{4(N-1)}\right)+\beta_{\varepsilon} R^{2}\right] .
$$

Для конформного скалярного поля $\left(\xi=\xi_{\text {c }}\right)$ при $N \rightarrow 4$ параметры $G_{\varepsilon}$ и $\beta_{\varepsilon}$ имеют конечную перенормировку (см. (9) и (18)). Но при $N \rightarrow 4$ перенормировка параметра $\alpha_{\varepsilon}$ в соответствии с $(12),(14)$ и $(40)$ бесконечна $\left(\sim(N-4)^{-1}\right)$ при любом значении константы ६ связи скалярного поля с кривизной.

\section{5. ЗАКЛЮЧЕНИЕ}

В данной работе исследовалась геометрическая структура контрчленов вакуумного ТЭИ скалярного поля с произвольной связью с кривизной в $N$-мерном однородном изотропном пространстве-времени. Полученные формулы (30), (31) определяют неперенормированные средние ТЭИ комплексного скалярного поля с произвольной связью с кривизной по вакууму, определяемому методом диагонализации гамильтониана [18]. Определена геометрическая структура контрчленов в методе эффективного действия для $N$-мерного однородного изотропного пространства (см. (8), (9), (18)). Дано обобщение $n$-волновой процедуры для однородных изотропных $N$-мерных пространств и определены соответствующие контрчлены (37), (38). Найдены свойства (см. (П.2)-(П.4)) собственных функций оператора Лапласа-Бельтрами в многомерных однородных изотропных пространствах, необходимые для вычисления вакуумного ТЭИ. Геометрическая структура контрчленов $n$-волновой процедуры проанализирована с помошью размерной регуляризации. Найдено аналитическое продолжение по размерности меры интегрирования в импульсном пространстве (см. (36), (П.9)). Показано совпадение геометрических структур первых трех вычитаний в $n$-волновой процедуре и в методе эффективного действия. Показано, что первые три вычитания (они исчерпьвают все вычитания в размерностях $N=4,5) n$-волновой процедуры в $N$-мерном однородном изотропном пространстве-времени соответствуют перенормировке космологической и гравитационной постоянных и параметров при квадратичных по кривизне слагаемых в затравочном гравитационном лагранжиане (41).

Сравнение вычитаний $n$-волновой процедуры (37), (38) при $N=4$ с контрчленами метода адиабатической регуляризации, приведенными в работе [9], показывает эквивалентность этих методов устранения расходимостей. Поэтому выводы о геометрической 
структуре вычитаний, сделанные в данной работе для $n$-волновой процедуры в четырехмерном однородном изотропном пространстве-времени, выполняются также и для метода адиабатической регуляризации.

ПРИЛОЖКЕНИЕ А

Приведем некоторые свойства полной ортонормированной системы собственных функций $\Phi_{J}(\mathbf{x})$ оператора Лапласа-Бельтрами $\Delta_{N-1}$ в $(N-1)$-мерном пространстве с метрикой $d l^{2}=\gamma_{\alpha \beta} d x^{\alpha} d x^{\beta}$ постоянной кривизны $K=0, \pm 1$, используемые при вычислении вакуумных средних ТЭИ. Обозначим

$$
f(\lambda)=\sum_{J(\lambda=\text { const })}\left|\Phi_{J}(\mathbf{x})\right|^{2},
$$

где $\lambda$ определено в (23) и для непрерывного $J$ суммирование заменяется интегрированием. В случае однородного изотропного пространства функция $f(\lambda)$ не зависит от $\mathbf{x}$. Применяя к (П.1) оператор $\Delta_{N-1}$ и учитывая (23), приходим к

$$
\sum_{J(\lambda=\text { const })} \gamma^{\alpha \beta} \partial_{\alpha} \Phi_{J}^{*}(\mathbf{x}) \partial_{\beta} \Phi_{J}(\mathbf{x})=\left(\lambda^{2}-\left(\frac{N-2}{2}\right)^{2} K\right) f(\lambda) .
$$

Из условия изотропии пространства с учетом (П.2) получим

$$
\sum_{J(\lambda=\text { const })} \partial_{\alpha} \Phi_{J}^{*}(\mathbf{x}) \partial_{\beta} \Phi_{J}(\mathbf{x})=\frac{\gamma_{\alpha \beta}}{N-1}\left(\lambda^{2}-\left(\frac{N-2}{2}\right)^{2} K\right) f(\lambda) .
$$

Применяя к (П.1) операции ковариантного дифференцирования $\widetilde{\nabla}_{\alpha} \widetilde{\nabla}_{\beta}$ в $(N-1)$-мерном пространстве и учитьвая (П.3), получим

$$
\sum_{J(\lambda=\text { const })}\left[\left(\widetilde{\nabla}_{\alpha} \widetilde{\nabla}_{\beta} \Phi_{J}^{*}\right) \Phi_{J}+\Phi_{J}^{*} \widetilde{\nabla}_{\alpha} \widetilde{\nabla}_{\beta} \Phi_{J}\right]=-\frac{2 \gamma_{\alpha \beta}}{N-1}\left(\lambda^{2}-\left(\frac{N-2}{2}\right)^{2} K\right) f(\lambda) .
$$

В квазиевклидовом случае $(K=0)$ в декартовых координатах

$$
\Phi_{J}(\mathbf{x})=(2 \pi)^{-(N-1) / 2} e^{-i \lambda_{\alpha} x^{\alpha}},
$$

где $-\infty<\lambda_{\alpha}<+\infty$. Интегрирование по сфере радиуса $\lambda$ в $(N-1)$-мерном пространстве "безразмерных импульсов" $\left\{\lambda_{\alpha}\right\}$ дает

$$
f(\lambda)=\frac{B_{N}}{2} \lambda^{N-2}, \quad K=0,
$$

где $B_{N}$ определено в разделе 3 .

В случае $K=1$ (сферическое пространство) при фиксированном $\lambda$ набор индексов $J$ конечньй и объем $(N-1)$-мерного пространства равен $S_{N}(1)$ - площади поверхности единичной сферы в $N$-мерном пространстве. Поэтому

$$
f(\lambda)=\frac{1}{S_{N}(1)} \int\left(\sum_{J(\lambda=\text { const })} \Phi_{J}^{*}(\mathbf{x}) \Phi_{J}(\mathbf{x})\right) \sqrt{\gamma} d^{N-1} \mathbf{x},
$$


где $\gamma=\operatorname{det}\left(\gamma_{\alpha \beta}\right)$. Меняя порядок операций суммирования и интегрирования и учитывая ортонормированность $\Phi_{J}(\mathbf{x})$, получим

$$
f(\lambda)=\frac{\operatorname{dim} \lambda}{S_{N}(1)}
$$

где $\operatorname{dim} \lambda$ - кратность собственного значения $\lambda^{2}-((N-2) / 2)^{2}$ оператора $-\Delta_{N-1}$. Используя соотношение, выражающее кратность собственных значений оператора Лапласа-Бельтрами на сфере через размерность пространства гармонических полиномов (см., например, [20]), приходим к следующей формуле:

$$
f(\lambda)=\frac{B_{N}}{2} \frac{\Gamma(n+N-2)}{\Gamma(n+1)} \lambda, \quad n=0,1, \ldots, \quad \lambda=n+\frac{N-2}{2} .
$$

Для произвольного $N$ и $K=0,1$ выражения (П.6), (П.7) могут быть записаны в виде

$$
f(\lambda)=\frac{B_{N}}{2}\left(\lambda^{N-2}+\alpha_{N} \lambda^{N-4}+\beta_{N} \lambda^{N-6}+\cdots\right)
$$

где

$$
\begin{aligned}
\alpha_{N} & =-\frac{1}{24}(N-2)(N-3)(N-4) K, \\
\beta_{N} & =\frac{1}{5760}(N-2)(N-3)(N-4)(N-5)(N-6)(5 N-8) K^{2} .
\end{aligned}
$$

ПРИЛОЖЕНИЕ Б

Приведем выражения для некоторых геометрических величин в $N$-мерном однородном изотропном пространстве-времени с метрикой (19).

Символы Кристоффеля, отличные от нуля:

$$
\Gamma_{00}^{0}=\frac{a^{\prime}}{a} \equiv c, \quad \Gamma_{0 \beta}^{\alpha}=c \delta_{\beta}^{\alpha}, \quad \Gamma_{\alpha \beta}^{0}=c \gamma_{\alpha \beta}, \quad \Gamma_{\beta \delta}^{\alpha}\left(g_{i k}\right)=\Gamma_{\beta \delta}^{\alpha}\left(\gamma_{\nu \mu}\right) .
$$

Компоненты тензора Риччи, отличные от нуля, и скалярная кривизна:

$$
\begin{gathered}
R_{00}=(N-1) c^{\prime}, \quad R_{\alpha \beta}=-\gamma_{\alpha \beta}\left[c^{\prime}+(N-2)\left(c^{2}+K\right)\right], \\
R=a^{-2}(N-1)\left[2 c^{\prime}+(N-2)\left(c^{2}+K\right)\right] .
\end{gathered}
$$

Компоненты тензора Эйнштейна:

$$
G_{00}=-\frac{(N-1)(N-2)}{2}\left(c^{2}+K\right), \quad G_{\alpha \beta}=\gamma_{\alpha \beta}(N-2)\left[c^{\prime}+\frac{N-3}{2}\left(c^{2}+K\right)\right] .
$$

Используя (П.10), (П.11), получим для квадрата тензора Риччи и вторых производных

$$
\begin{gathered}
R_{l m} R^{l m}=a^{-4}(N-1)\left[N c^{2}+2(N-2) c^{\prime}\left(c^{2}+K\right)+(N-2)^{2}\left(c^{2}+K\right)^{2}\right], \\
\nabla_{0} \nabla_{0} R=2 a^{-2}(N-1)\left[c^{(3)}+(N-7) c^{\prime \prime} c+(N-4) c^{2}-\right. \\
\left.\quad-6(N-3) c^{\prime} c^{2}+3(N-2) c^{4}+K(N-2)\left(3 c^{2}-c^{\prime}\right)\right], \\
\nabla_{\alpha} \nabla_{\beta} R=2 \gamma_{\alpha \beta} a^{-2}(N-1)\left[-c^{\prime \prime} c-(N-4) c^{\prime} c^{2}+(N-2) c^{2}\left(c^{2}+K\right)\right], \\
\nabla^{l} \nabla_{l} R=2 a^{-4}(N-1)\left[c^{(3)}+2(N-4) c^{\prime \prime} c+(N-4) c^{2}+\right. \\
\left.+\left(N^{2}-10 N+20\right) c^{\prime} c^{2}-(N-2)\left((N-4) c^{2}+c^{\prime}\right)\left(c^{2}+K\right)\right] .
\end{gathered}
$$


Используя (П.11), (П.13), для компонент ${ }^{(1)} H_{i k}$ и ${ }^{(3)} H_{i k}($ см. $(13),(17))$ получим

$$
\begin{aligned}
{ }^{(1)} H_{00}= & \frac{(N-1)^{2}}{a^{2}}\left[2 c^{\prime 2}-4 c^{\prime \prime} c-4(N-4) c^{\prime} c^{2}-\frac{c^{4}}{2}(N-2)(N-10)-\right. \\
& \left.-K(N-2)\left(\frac{N-2}{2} K+(N-6) c^{2}\right)\right], \\
{ }^{(1)} H_{\alpha \beta}= & \gamma_{\alpha \beta} a^{-2}(N-1)\left\{4 c^{(3)}+4(2 N-9) c^{\prime \prime} c+2(3 N-11) c^{\prime 2}+\right. \\
& +6\left(N^{2}-10 N+20\right) c^{\prime} c^{2}+\frac{1}{2}(N-2)\left(N^{2}-15 N+50\right) c^{4}+ \\
& \left.+K(N-2)\left[(N-5)(N-6) c^{2}+2(N-6) c^{\prime}+\frac{1}{2}(N-2)(N-5) K\right]\right\}, \\
{ }^{(3)} H_{00}= & \frac{1}{2} a^{-2}(N-1)(N-2)(N-3)\left(c^{2}+K\right)^{2}, \\
{ }^{(3)} H_{\alpha \beta}= & -\frac{1}{2} \gamma_{\alpha \beta} a^{-2}(N-2)(N-3)\left[4 c^{\prime}\left(c^{2}+K\right)+(N-5)\left(c^{2}+K\right)^{2}\right] .
\end{aligned}
$$

Благодарности. Автор благодарен проф. А. А. Грибу за обсуждение работы. Работа поддержана Министерством образования РФ (гранты № Е00-3-163 и Е02-3.1-198).

\section{Список литературы}

[1] А. А. Гриб, С. Г. Мамаев, В. М. Мостепаненко. Вакуумные квантовые эффекты в сильных полях. М.: Энергоатомиздат, 1988.

[2] Н. Бирелл, П. Девис. Квантованные поля в искривленном пространстве-времени. М.: Мир, 1984.

[3] A. A. Grib, Yu. V. Pavlov. Int. J. Mod. Phys. D. 2002. V. 11. P. 433; Int. J. Mod. Phys. A. 2002. V. 17. P. 4435.

[4] L. Parker, A. Raval. Phys. Rev. D. 1999. V. 60. P. 063512; Phys. Rev. Lett. 2001. V. 86. P. 749.

[5] Я. Б. Зельдович, А. А. Старобинский. ЖЭТФ. 1971. Т. 61. С. 2161.

[6] L. Parker, S. A. Fulling. Phys. Rev. D. 1974. V. 9. P. 341.

[7] M. Bordag, J. Lindig, V. M. Mostepanenko. Class. Q. Grav. 1998. V. 15. P. 581.

[8] S. Habib, C. Molina-París, E. Mottola. Phys. Rev. D. 2000. V. 61. P. 024010.

[9] T. S. Bunch. J. Phys. A. 1980. V. 13. P. 1297.

[10] С. Г. Мамаев, В. М. Мостепаненко, В. А. ШШелюто. ТМФ. 1985. Т. 63. № 1. С. 64.

[11] Ю. В. Павлов. ТМФ. 2001. Т. 128. № 2. С. 236.

[12] N. A. Chernikov, E. A. Tagirov. Ann. Inst. H. Poincaré. A. 1968. V. 9. P. 109.

[13] T. S. Bunch. J. Phys. A. 1979. V. 12. P. 517.

[14] G. 't Hooft, M. Veltman. Nucl. Phys. B. 1972. V. 44. P. 189.

[15] Б. С. де Витm. Динамическая теория групп и полей. М.: Наука, 1987.

[16] В. Л. Гинзбург, Д. А. Киржнич, А. А. Любушин. ЖЭТФ. 1971. Т. 60. С. 451.

[17] С. Г. Мамаев, В. М. Мостепаненко, А. А. Старобинский. ЖЭТФ. 1976. Т. 70. С. 1577.

[18] Ю. В. Павлов. ТМФ. 2001. Т. 126. № 1. С. 115.

[19] B. S. Dewitt. Phys. Rep. C. 1975. V. 19. P. 295; B. C. де Витm. Квантовая теория поля в искривленном пространстве-времени. В сб.: Черные дыры. М.: Мир, 1978. С. 66.

[20] M. А. Шубин. Псевдодифференциальные операторы и спектральная теория. М.: Наука, 1978.

Поступила в редакцию 5.VI.2002 г., после доработки 20.III.2003 г. 